absence of GR ligands, GR is transcriptionally activated via p38MAPK-dependent phosphorylation of Ser134 upon exposure of TNBC cells to TME-derived agents (TGF $\beta$, HGF). The ligandindependent pS134-GR transcriptome primarily encompasses gene sets associated with TNBC cell survival and migration/invasion. Accordingly, pS134-GR was essential for TGF $\beta$-induced TNBC cell migration, anchorage-independent growth in soft-agar, and tumorsphere formation, an in vitro readout of breast cancer stemness properties. Finally, a 24-gene pSer134-GR-dependent signature induced by TGF $\beta 1$ predicts shortened survival in breast cancer. We expect to find similar results using an in-house tissue microarray. DISCUSSION/SIGNIFICANCE OF IMPACT: Phospho-S134-GR is a critical downstream mediator of p38 MAPK signaling and TNBC migration, survival, and stemness properties. Our studies define GR as a required effector of TGF $\beta 1$ signaling and nominate $\mathrm{pS} 134-\mathrm{GR}$ as a biomarker of elevated risk of breast cancer dissemination.

4241

\section{Identification of small molecules that facilitate the efficient differentiation of stem cell derived $\beta$-cells ${ }^{*}$ Yuhao Min ${ }^{1}$, Chris Clifford, and Quinn P. Peterson \\ ${ }^{1}$ Mayo Clinic}

OBJECTIVES/GOALS: In this study, we established a highthroughput chemical screening platform to identify small molecules that facilitates efficient differentiation of stem cells derived $\beta$ (SC- $\beta$ ) cells. Using this platform, we identified several compounds that potentially increase the differentiation efficiency. METHODS/ STUDY POPULATION: Differentiation of human embryonic stem cells (HUES8) into SC- $\beta$ was carried out using previously published protocols in a $3 \mathrm{D}$ cell suspension. Single cells were replated in Matrigel-coated well plates at the start of different stages depending on experiments. Differentiation medium supplemented with small molecules at a final concentration of $2 \mathrm{M}$ and $0.2 \mathrm{M}$ was used throughout the stage. All the cells were then fixed and permeabilized. Immunocytochemical staining was performed. Images of each well were taken and analyzed. Numbers of the total cell, insulinpositive cell, NKX6.1-positive cell, and co-positive cell were recorded. Candidate compounds were validated using flow cytometry or ICC. RESULTS/ANTICIPATED RESULTS: We identified several hit compounds that significantly increase the NKX6.1 positive cell percentage compared to the DMSO-treated controls when treated at the PP1 cell stage. Follow up assays demonstrated that at least one of these putative hits reproducibly increased NKX6.1 expression. In addition, we identified other compounds that significantly increase the insulin and NKX6.1 copositive SC- $\beta$ cell population when treated at the later PP2 cell stage during the differentiation. We expect a dosage-dependent response when the candidate hits are validated using more accurate assays. DISCUSSION/SIGNIFICANCE OF IMPACT: We established a high-throughput screening platform to identify small molecules that increase the efficiency of SC- $\beta$ direct differentiation. Successful generation of SC- $\beta$ allows cell replacement therapy in diabetes patients, and a better understanding of pancreatic biology and development.
Impaired Natural Killer Cell Function May Be Associated with Cancer-related Fatigue

Jeniece Regan ${ }^{1}$, Rebekah Feng, and Leorey Saligan

${ }^{1}$ National Institutes of Health

OBJECTIVES/GOALS: During and after cancer treatment, cancerrelated fatigue $(\mathrm{CRF})$ is a debilitating symptom reported by up to $80 \%$ of cancer patients Our understanding of the pathology underlying $\mathrm{CRF}$ is limited. Preliminary RNA sequencing data suggest that increased levels of KIR3DL1, the natural killer cell (NK) immunoglobulin-like receptor 3DLla, may be associated with CRF. METHODS/STUDY POPULATION: Fatigue was measured using the Functional Assessment of Chronic Illness Therapy-Fatigue (FACT-F). Functional validation of the NK cell finding was performed from whole blood obtained from fatigued and non-fatigued subjects. NK cells were isolated from freshly collected whole blood using a human NK cell isolation kit based on CD56 microbead positive selection. NK cell function was assessed using the NK cell direct cytotoxicity assay. Briefly, isolated NK cells were co-cultured in a 2:1 ratio with calcein AM-labelled K562 cells, which are NK cell-sensitive due to the very low MHCI expression. NK cell-mediated cytotoxicity was assessed with Cytation 1 Cell Imaging Multi-Mode reader. Flow cytometric protocols were used to examine NK subset differences between the fatigued and non-fatigued groups. RESULTS/ANTICIPATED RESULTS: NK cells isolated from the fatigued group exhibited decreased cytotoxicity at $12.28 \%$ compared to NK cells isolated from non-fatigued controls at a mean of $40.6 \%$ cytotoxicity. Flow cytometry analysis revealed a decrease in the $\mathrm{CD} 56^{\mathrm{dim}} \mathrm{CD} 16^{\text {bright }}$ population in the fatigued group (87.1\% of CD56+CD4- cells) compared to the control $(91.4 \%$ of CD56+CD4- cells). Furthermore, there was a decrease in NKG2A expression in mature NK cells $\left(\mathrm{CD} 56^{\text {dim }} \mathrm{CD} 16^{\text {bright }}\right.$ ) isolated from the fatigued group compared to the non-fatigued group. DISCUSSION/SIGNIFICANCE OF IMPACT: Results from the pilot study suggest that there was a decrease in NK cell cytotoxicity in the fatigued group. In addition, there may be a shift in NK cell subpopulations associated with fatigue. Findings from this pilot study suggest that impaired NK cell function may be associated with CRF pathogenesis.

4323

\section{Inflammation partially mediates fatigue-like behavior in mice}

Sarah Alshawi ${ }^{1}$, Brian Wolff ${ }^{1}$, and Leorey Saligan ${ }^{1}$

${ }^{1}$ National Institutes of Health

OBJECTIVES/GOALS: Fatigue is a distressing side effect of cancer and its treatment. It is a subjective symptom that can include mental, physical, emotional, and motivational components. We sought to determine whether preventing inflammation affects fatigue-like behavior in a mouse model of radiation therapy. METHODS/ STUDY POPULATION: C57BL/6 mice received three consecutive 8 gray doses of daily peripheral irradiation. We used voluntary wheel running activity to measure fatigue-like behavior before and after this period. Minocycline, an antibiotic with anti-inflammatory effects, was administered beginning a week before irradiation and 
continued throughout the experiment. We also tested mice lacking the toll-like receptor adaptor protein, MyD88. Cognitive abilities were tested using spontaneous alternation in a Y-maze. RESULTS/ ANTICIPATED RESULTS: We found that minocycline reduces fatigue-like behavior exhibited after irradiation, but had no effect on pre-irradiation activity levels. Similarly, fatigue-like behavior after radiation was partially reversed by genetic loss of MyD88. Y-maze spontaneous alternation performance remained similar in all groups. DISCUSSION/SIGNIFICANCE OF IMPACT: Both pharmacological and genetic anti-inflammatory manipulations increased voluntary activity levels after irradiation. Our results suggest that inflammation is an important factor in the development of fatigue-like behavior. Modulators of inflammatory processes hold potential for alleviating fatigue.

4361

\section{Interim Results of the Effect of Haptoglobin Phenotype on Inflammatory Cytokine Concentrations in Plasma and CSF After Aneurysmal Subarachnoid Hemorrhage \\ Austin Smith ${ }^{1}$ \\ ${ }^{1}$ University of Kentucky Center for Clinical and Translational Science}

OBJECTIVES/GOALS: Haptoglobin (Hp) phenotypes may affect inflammatory response after neurologic injury. We are investigating the relationship between patient $\mathrm{Hp}$ phenotypes and inflammatory cytokine concentrations in plasma and CSF after aneurysmal subarachnoid hemorrhage (aSAH), a severe form of hemorrhagic stroke. METHODS/STUDY POPULATION: Following IRB approval, all patients with angiographically-proven aSAH and who underwent extraventricular drain (EVD) placement were included. Patients were excluded if they were not expected to survive hospitalization or were on pre-existing anti-inflammatory agents. For all enrolled patients, plasma and CSF samples were taken on post-bleed days $3,5,7$, and 10 , processed and then frozen for later analysis. In this interim analysis, Hp phenotype was assessed through ELISA analysis of plasma samples and cytokine concentrations were determined using multiplex ELISA kits for both plasma and CSF samples. Hp phenotypes were dichotomized to either HP1 (Hp 1-1 or 1-2) or HP2 (Hp 2-2). RESULTS/ANTICIPATED RESULTS: To date, 23 aSAH patients have been enrolled in this IRB-approved study. An interim analysis of the first 13 patients has revealed eight Hp1 patients (Hp 1-1 n = 1, Hp 1-2 n=7) and five Hp2 patients. CSF levels of IL-6 and TNF-a were greater than plasma levels in all patients at all time points. CSF levels of IL-6 appear to peak on PBD $5(1890 \pm 767 \mathrm{pcg} / \mathrm{mL})$ and $7(1612 \pm 899 \mathrm{pcg} / \mathrm{mL})$. The CSF IL-6 concentrations in the Hp2 group were lower (1792 \pm $806 \mathrm{pcg} / \mathrm{mL}$ vs. $1952 \pm 791 \mathrm{pcg} / \mathrm{mL})$ on PBD5 but were higher $(1635 \pm 930 \mathrm{pcg} / \mathrm{mL}$ vs $1598 \pm 943 \mathrm{pcg} / \mathrm{mL})$ at PBD7; however, these differences did not reach statistical significance. DISCUSSION/ SIGNIFICANCE OF IMPACT: This interim analysis demonstrated no statistically significant differences in plasma or CSF cytokine concentrations between patients with different $\mathrm{Hp}$ phenotypes. This may be due to the low number of samples or the potential confounding effect of disease-specific secondary neurological injuries.
4126

Intermuscular adipose tissue secretes pro-inflammatory, extracellular matrix, and lipid signals related to insulin resistance and type 2 diabetes

Darcy Kahn ${ }^{1}$, Simona Zarini ${ }^{2}$, Emily Macias ${ }^{2}$, Amanda Garfield ${ }^{2}$, Kathleen Harrison ${ }^{2}$, Melanie Cree-Green ${ }^{2}$, Jonathan Schoen ${ }^{2}$, and Bryan Bergman ${ }^{2}$

${ }^{1}$ University of Colorado at Denver; ${ }^{2}$ University of Colorado, Anschutz Medical Campus

OBJECTIVES/GOALS: Intermuscular adipose tissue (IMAT) has been associated with insulin resistance and type 2 diabetes, yet mechanistic studies addressing the functional role of IMAT are lacking. The aim of this work was to identify novel mechanisms by which IMAT may directly impact skeletal muscle metabolism. METHODS/ STUDY POPULATION: We quantified the secretome of IMAT, subcutaneous adipose tissue (SAT), and visceral adipose tissue (VAT) to determine if there are differences between depots in the secretion of cytokines, eicosanoids, FFAs and proteins that influence metabolic function. SAT and VAT biopsies from patients undergoing laparoscopic bariatric surgery and IMAT extracted from vastus lateralis biopsies of individuals with Obesity were cultured for 48 hours in DMEM, and the conditioned media was analyzed using nanoflow HPLC-MS, multiplex ELISAs and LC/MS/MS for proteins, cytokines and eicosanoids/FFA, respectively. RESULTS/ ANTICIPATED RESULTS: IMAT secretion of various extracellular matrix proteins (fibrinogen- $\beta$, collagenV1a3, fibronectin) was significantly different than VAT and SAT. Pro-inflammatory cytokine secretion of IFNg, TNFa, IL-8 and IL-13 from IMAT was higher than VAT and significantly higher than SAT $(p<0.05)$. IMAT secretes significantly more pro-inflammatory eicosanoids $\mathrm{TXB}_{2}$ and $\mathrm{PGE}_{2}$ than VAT $(p=0.02,0.05)$ and SAT $(p=0.01,0.04)$. IMAT and VAT have significantly greater basal lipolysis assessed by FFA release rates compared to SAT $(\mathrm{p}=0.01,0.04)$. DISCUSSION/ SIGNIFICANCE OF IMPACT: These data begin to characterize the disparate secretory properties of SAT, VAT and IMAT and suggest a metabolically adverse secretome of IMAT, that due to its proximity to skeletal muscle may play an important functional role in the pathogenesis of insulin resistance and type 2 diabetes.

4558

Investigating the functional consequences of anaplastic lymphoma kinase (ALK) mutations arising upon Lorlatinib treatment

Gabriela Maria Witek ${ }^{1}$, Whelton Miller, David Slochower, Esther Berko, Yael Mossé, Mark Lemmon, and Ravi Radhakrishnan

${ }^{1}$ University of Pennsylvania School of Medicine

OBJECTIVES/GOALS: Neuroblastoma (NB) is an embryonal cancer of the sympathetic nervous system that affects mostly infants and young children. The complex genetic background present across NB patients results in diverse clinical response and difficulty in individualizing therapy. Currently, NB patients undergo a regimen of genotoxic chemotherapeutics, radiation therapy, and new immunotherapy that, while effective, has significant side effects, including excruciating pain. One promising avenue for targeted therapy in neuroblastoma focuses on anaplastic lymphoma kinase (ALK), a cell surface neural receptor tyrosine kinase. We previously identified 